\title{
Phylostratigraphic approach in evolutionary analysis: comparison of methods
}

\author{
Tatiana Martusheva \\ Novosibirsk State University \\ NSU, Novosibirsk, Russia \\ t.maryanovskaya@g.nsu.ru
}

\author{
Zakhar Mustafin \\ Kurchatov Genomics Center, \\ ICG SB RAS, Novosibirsk, Russia \\ mustafinzs@bionet.nsc.ru
}

\author{
Sergey Lashin \\ Kurchatov Genomics Center, \\ ICG SB RAS, Novosibirsk, Russia \\ NSU, Novosibirsk, Russia \\ lashin@bionet.nsc.ru
}

\begin{abstract}
Phylostratigraphic analysis allows us to determine the "age of the gene" - evolutionary time of its origin. There are different methods to calculate this age, each of which has its own issues. In this work, we compare several studies on phylostrastigraphic analysis. The correlation coefficient of the results of two research with different phylostratigraphic trees but the same method to count phylostratigraphic age (BLAST-based) was found to be 0.76 . Comparison of the results of calculating the phylostratigraphic age by Orthoscape application (used KEGG as data source) and the myTAI package (used BLAST as data source) showed a lower corellation coefficient - only 0.27. It is planned to continue the studying of differences in the results of different methods and their reasons.
\end{abstract}

Key words - phylostratigraphy; evolution; gene age; BLAST; KEGG; ortholog

\section{Motivation and Aim}

Phylostratigraphic analysis is a promising field of evolutionary analysis that allows us to determine the most important steps in the evolution of organisms [1]. Using this analysis, one can determine the "age of the gene" - the presumed stage of the origin of the gene in the process of evolution of the organism. The age of a gene is based on the analysis of orthologous genes and the search for the last common ancestor for the gene and its orthologs on the evolutionary tree. There are several ways to search for orthologous genes and their positions on that tree, the most popular of which is using the Basic Local Alignment Search Tool, https://blast.ncbi.nlm.nih.gov/Blast.cgi). Though this method is effective, however, it has some drawbacks [2], [3], which encourages the use of other tools. In particular, databases on orthologous genes can be used, for example, KEGG (Kyoto Encyclopedia of Genes and Genomes, https://www.kegg.jp) and EggNOG (http://eggnogdb.embl.de/\#/app/home). In this study, we compare the results of using different methods underlying phylostratigraphic analysis.

\section{Methods and Algorithms}

We have reviewed scientific papers with published data on the analysis of gene age, as well as programs for this analysis. One of these programs is Orthoscape [4], which is based on the KEGG orthologs database. Yet another phylostratigraphic information was taken from phylotranscriptomic studies in particular used myTAI package [5], which defines orthologs and position on the taxonomic tree using BLAST. Phylostratigraphic ages resulted from the programs mentioned above, as well as taken from published papers, were compared, in particular, in terms of correlation. We have additionally reduced all the taxonomy data to the uniform tree, which allowed us to make such a meta-analysis.
Results

We have compared phylostratigraphic ages indexes of Arabidopsis thaliana genes taken from the publications of Ruprecht and co-authors [6] and Quint and co-authors [7]. Both studies used BLAST, but the authors took different taxonomic trees represented key evolutionary points of plants. Correlation coefficient for initial trees was shown to be 0.74 as well as 0.76 for the uniform trees. This suggests that the selection of key taxa in the phylostratigraphic tree did not significantly affect the age of genes when analyzed using the same method. Comparison of the results of calculating the philostratigraphic age of genes using the Orthoscape application, which uses the KEGG database as a source of data on orthologous genes, and the myTAI package, which uses BLAST, showed a significantly lower correlation coefficient - only 0.27 , which indicates a fundamental difference in the result when using these methods. Orthoscape found significantly more young genes, while myTAI found older ones. This is due to separate groups of genes that differ significantly in age in different methods, for example, ribosomal genes are as old as possible in the first method, and as young as possible in the second one. This may also be due to fundamentally different parameters for selecting orthologs, in particular, the minimum identity of amino acid sequences of proteins encoded by genes, which is necessary for genes to be considered orthologs. In the future, this issue will be studied in more detail and various options for selecting orthologs will be considered and the results obtained will be compared.

\section{Acknowledgment}

The study is supported by RFBR, grant number 20-0400885 A.

\section{References}

[1] T. Domazet-Lošo et al. A phylostratigraphy approach to uncover the genomic history of major adaptations in metazoan lineages, Trends Genet., vol. 23, no. 11, 2007, doi: 10.1016/j.tig.2007.08.014.

[2] L. B. Koski and G. B. Golding, The Closest BLAST Hit Is Often Not the Nearest Neighbor, J. Mol. Evol., vol. 52, no. 6, pp. 540-542, Jun. 2001, doi: 10.1007/s002390010184.

[3] B. A. Moyers and J. Zhang, Phylostratigraphic Bias Creates Spurious Patterns of Genome Evolution, Mol. Biol. Evol., vol. 32, no. 1, pp. 258-267, Jan. 2015, doi: 10.1093/molbev/msu286.

[4] Z.S. Mustafin et al. Orthoscape: a cytoscape application for grouping and visualization KEGG based gene networks by taxonomy and homology principles, BMC Bioinformatics, vol. 18, no. S1, pp. 1-9, 2017, doi: 10.1186/s12859-016-1427-5.

[5] H.-G. Drost et al. myTAI: evolutionary transcriptomics with R, Bioinformatics. 2018, doi: 10.1093/bioinformatics/btx835.

[6] C. Ruprecht et al. Phylogenomic analysis of gene co-expression networks reveals the evolution of functional modules, Plant J. 2017, doi: 10.1111/tpj.13502.

[7] M. Quint et al. A transcriptomic hourglass in plant embryogenesis, Nature. 2012, doi: 10.1038/nature11394. 